\title{
FLIGHT TESTS OF RANGES AND LATENCIES OF A THREEFOLD REDUNDANT C2 MULTI-LINK SOLUTION FOR SMALL DRONES IN VLL AIRSPACE
}

\author{
Andreas Volkert \\ Institute of Flight Guidance \\ DLR (German Aerospace Center) \\ Braunschweig, Germany \\ Helge Hackbarth \\ Kopterkraft \\ Tallinn, Estonia \\ Teemu Joonas Lieb, Stefan Kern \\ Institute of Flight Guidance \\ DLR (German Aerospace Center)
}

Braunschweig, Germany

\begin{abstract}
Over the past years the UAV market has grown rapidly and will continue so. Several manufacturer offer UAVs, which differ in their size, equipment or performance. Besides the basic regulations many initiatives deal with the question of how a future airspace management system for unmanned and manned airspace users could look like.
\end{abstract}

The German Aerospace Center (DLR) has started the City-ATM project [1], which seeks to identify essential components for such a system. The focal point is on a safe, secure and efficient integration of new airspace users, but also to be robust against failures. Therefore the drone operator needs on the one hand information about his drone and on the other hand information about the surrounding airspace users.

In the course of City-ATM, DLR works together with several external partners to find, test and validate a variety of solutions which enable such an airspace management system as well as necessary sub-systems and solutions that are needed in such a system. One of the solutions to find and validate is a redundant communication system to enable reliable data exchange between drone and pilot as well as unmanned aerial system and airspace management system.

In this paper the technical setup, consisting of a (mobile) ground and air module and the basic functionality will be described to meet the requirements from a future UTM system. In addition the HyraCom system has been evaluated by conducting flight tests under real conditions and external influences in VLL (Very Low Level) airspace. In this context communication range and latency of each $\mathrm{C} 2$ link has been used as performance criteria.

\section{Introduction}

Small drones are getting more and more in the skies. The primary airspace they are foreseen to be used in is the Very Low Level (VLL) airspace. With a rising number of drones the situational awareness of a drone pilot becomes even more important. The drone pilot has to be aware of the status of his drone, of the surrounding traffic and his control and command (C2) inputs must be delivered from the 
Ground Control Station (GCS) to the drone. But also other participants have to be aware of the small drone, especially where it is and what it is. A future UTM System or European U-space System will incorporate a tracking service, which gathers all data from drones in VLL airspace and provides them to the drone pilot or other users. Such a system needs information of the drone's GPS coordinates and identification code. The prominent flight controller developer PX4 just released version 1.8.2 [3], which is able to extract the so called UTM_GLOBAL_POSITION from the Pixhawk flight controller which contains all this information. The tracking service of the U-space system is very important, since a lot of other U-space Services (Monitoring, Emergency Management, Density Management, Deconfliction, etc.) are dependent on it and it provides the best situational awareness for a drone pilot. The tracking service in turn relies on data from the drones, thus a stable, secure and reliable data link is required to transmit data with low latency. Thus, in this paper, the maximum range and latencies of a $\mathrm{C} 2$ link for small drones shall be tested.

Nowadays there are a number of manufacturers who offer such datalinks, but mostly these are limited to operations in Visual Line of Sight (VLOS) or Radio Line of Sight (RLOS). Some manufacturer already offer a $4 \mathrm{G}$ LTE data link which is able to use multiple networks from different service providers, which is a good step to operations Beyond Visual Line of Sight (BVLOS) and Beyond Radio Line of Sight (BRLOS), but still are limited to 4G LTE coverage.

In the course of the City-ATM project, KopterKraft [2] has developed a three-time redundant communication network between drone operator and the drone, which is AES (Advanced Encryption Standard) encrypted. It has the ability to share its position with others by sending it to a UTM tracking service as well as the German air navigation service provider (DFS). Based on 4G LTE, $2.4 \mathrm{GHz}$ and 868 $\mathrm{MHz}$ the multi-radio solution (HyraCom) provides an opportunity for safe operation in rural areas, where LTE is less available compared to urban areas. In case of insufficient 4G LTE coverage or intensity, the radio links will be in charge to exchange the data between drone and GCS. Therefore the whole system is threefold redundant.
Furthermore the developed solution offers mesh capability to get an even further communication range, if additional intermediate radio modules are used. Usually the radio links are limited to their signal strength to work properly, but if a repeater is used to receive, amplify and transmit the signal, the range can be extended. When a repeater is connected to the radio link, it is called a mesh network in this paper.

The tests were done in Hamburg/Germany for an urban environment and on a temporary inoperative airport in Cochstedt/Germany for a rural environment.

\section{Related Work}

\section{City-ATM}

Within the City-ATM project, DLR and several Stakeholders (NXP, KopertKraft, FlyNex, Auterion, DFS and ZAL) develop an overall concept for future air traffic management (ATM) in urban airspace, which will enable safe and efficient integration of new airspace users (such as unmanned aerial vehicles and air taxis) [4]. Aside from general requirements analysis the project deals with the definition and validation of operational and technical concepts. This includes airspace management, information provision, traffic flow control and monitoring, as well as CNS-infrastructure. To evaluate these concepts at different development stages, a simulation and demonstration platform for urban ATM is developed. This architecture consists of different airspace management services and tools, connected with each other and allowing all stakeholders assessments on feasibility of new concept elements, by using realistic use cases and flight tests.

\section{CORUS}

CORUS (Concept of Operation for European UAS Systems) defines a ConOps for unmanned aircraft system in very low level airspace and provides the foundation for European Unmanned aircraft system Traffic Management (UTM) systems. CORUS is an overarching project for several other UAS projects and sets a baseline for almost all of them. In the ConOps three airspace "colors" are described including rules of the air, services and obligations. Red airspace can only be entered with specific permission. Green airspace allows easy 
access but requires the drone pilot to maintain separation by maintaining visual contact with the drone at all times (VLOS). And there is Amber airspace introduced at $\mathrm{U} 2$ level [5] which provides strategic conflict resolution, but at a cost; access to this airspace requires a flight plan and tracking for all drones; even those flying in VLOS.

Tracking drones will require that they (or something they are carrying) emit position reports and that these find their way back to a U-space service that is creating a track (Tracking Service). This could occur in numerous ways, but CORUS does not specify how. The system which is tested in this paper is able to reports its position (UTM_GLOBAL_POSITION) via 4G LTE to the World Wide Web.

\section{$D L R$}

The institute of communication and navigation of DLR investigated the requirements for communication links and identified the required messages which have to be transmitted. They found that the level of autonomy influences the communication link utilization and therefore increases the requirements on such a link [6].

The institute of flight guidance investigates a density-based management concept for urban air traffic in U-space, where a safety ellipsoid is assigned to each airspace users. The size of this ellipsoid is depending on navigation, communication and the capability to detect other airspace users (cooperatively and uncooperatively). For communication parameters such as datalink latency, robustness, integrity and availability define specific parameters describing the ellipsoidal shaped bound for each aircraft [7].

Furthermore the institute of flight guidance is testing a communication system for air-to-air and airto-ground intercommunication of UAVs. The D2X (Drone-to-X) technology is derived from the V2X technology used in cars. D2X uses a $5.8 \mathrm{GHz}$ radio link for communication with ground peers in the vicinity and/or other drones or airspace users equipped with D2X. Tested with its legal transmitting sensitivity it achieves a moderate range but offers sufficient bandwidth for data exchange such as cooperative collision avoidance maneuvers [8].

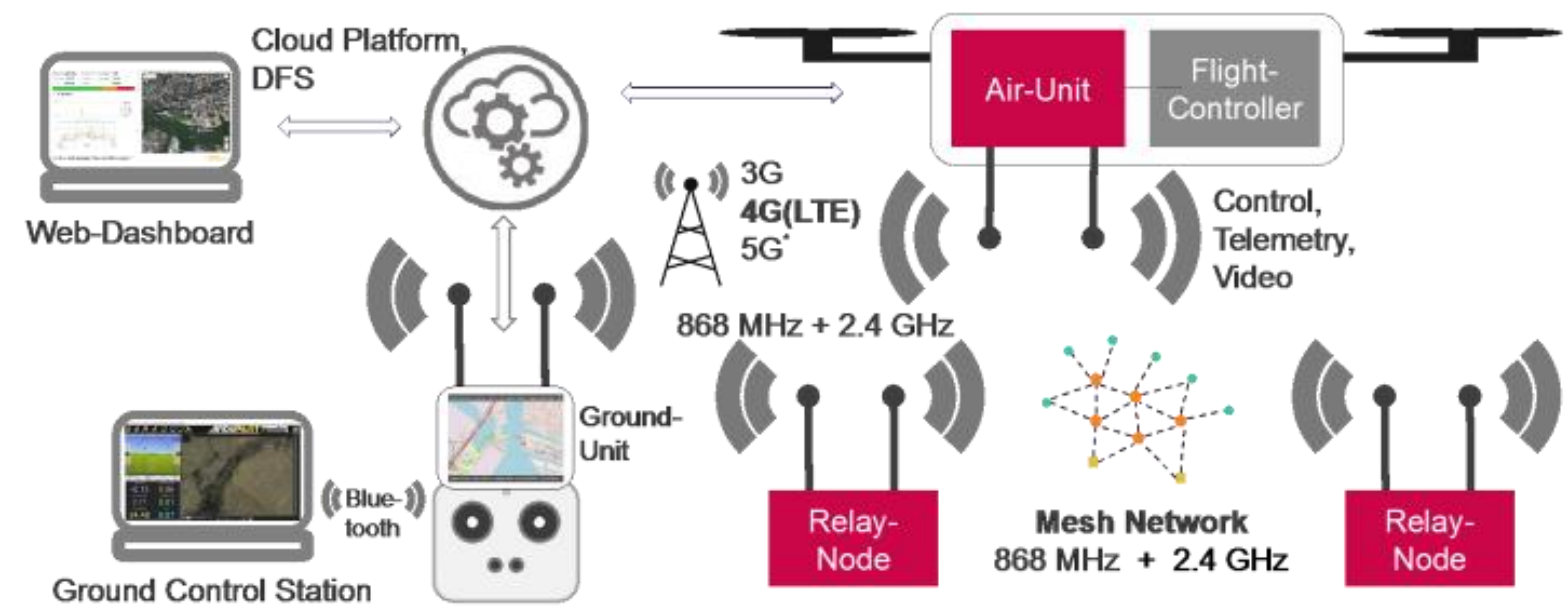

Figure 1 - Triple redundant BVLOS RC-/Data Communication 


\section{System Architecture}

\section{Communication Scenario}

HyraCom implements a triple redundant radio communication. The primary connection is using a mobile network link (3G / 4G (LTE) and - if available - 5G). A $2.4 \mathrm{GHz}$ and an $868 \mathrm{MHz}$ longrange connection with ad-hoc mesh network capability act as fallback when the drone or the pilot moves into a location without mobile network (refer to Figure 1). The tested multi-link system transmits:

- 16 remote control channels from the pilot's hand controller to the aircraft

- transparent serial data link provided as serial UART / Bluetooth virtual COM port on ground- and air-unit. This link is typically used for telemetry and additional mission control (waypoint missions etc.)

- optionally: secondary bidirectional high speed data link (only via LTE) e.g. for video and custom data

- optionally: mission status like position data, height, velocity, direction, aircraft ID and real time condition data to a cloud service as well as to the DFS (German air-traffic control). Currently this information is based on the MAVLink message type UTM_GLOBAL_POSITION.

- optionally: Broadcasts messages injected from relay nodes

\section{Package management}

Control and data packages are sent through all three radio links in parallel. Data from the packages which arrives first on the other end will be forwarded to the output; subsequent packages with the same (or older) timestamp arriving via other channels will be ignored. This makes sure that latency is minimized dynamically - ,first come, first taken“.

As bandwidth on $868 \mathrm{MHz}$ and $2.4 \mathrm{GHz}$ radio is limited in favor of longer transmission ranges, there is some additional traffic shaping applied to the data if HyraCom detects MAVLink packages on the telemetry stream. This reduction does not affect the LTE mobile network link.

\section{Range Extension through Mesh Networks}

In order to remotely control BVLOS (beyond visual line of sight) missions with almost unlimited operation range, most drone communication systems are primarily based on communication through the mobile network infrastructure $(3 \mathrm{G}, 4 \mathrm{G}, 5 \mathrm{G})$. But this infrastructure is not always and everywhere guaranteed or high latencies may occur when traveling between network cells (mobile network prioritizes packages from voice communication higher than data packages), HyraCom backs up communication through two additional radio links on $2.4 \mathrm{GHz}$ and $868 \mathrm{MHz}$ (in other countries outside Europe, $915 \mathrm{MHz}$ modules may be used instead).

As transmission power is quite limited in Europe on these free ISM bands (HyraCom respects these limits) it supports a range extension using additional relay nodes (refer to Figure 2). These nodes automatically setup a Xbee / IEEE 802.15.4 based mesh network supporting $n: m$ communication (not only peer-to-peer) so that data packages will even be forwarded to end nodes which would be outside transmission range or disturbed by objects (buildings, trees etc.) within the visual line of sight between ground unit and aircraft. It also allows you to setup your own communication infrastructure across ranges which have no mobile network coverage. The relay nodes can also be used to send broadcast messages to all other nodes.

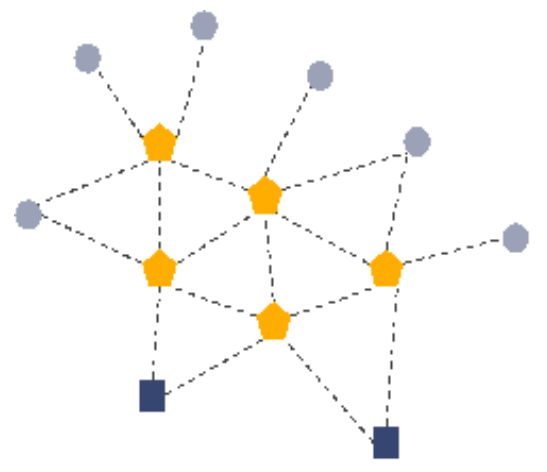

Figure 2 - Mesh network consisting of gateways, repeaters and endpoints increase range and reliability 


\section{Security, Robustness and Fairness}

HyraCom's communication uses AES (Advanced Encryption Standard) encryption on the radio links and packages carry additional checksums for error detection. This makes it impossible for third parties to lurk or hack into the communication in order to take over the aircraft. As it will require disturbing all three communication links at the same time, effort to disturb the connection to the aircraft is extremely high.

In order to not be restricted by duty cycle limitations, the $868 \mathrm{MHz}$ communication implements interference reduction techniques like LBT (listen before talk) and AFA (adaptive frequency agility). This allows others to use the limited resources of the ISM bands as postulated by ETSI (European Telecommunications Standards Institute) / Federal Network Agency [9].

\section{Ground Unit Architecture}

The microcontroller ensures real-time capability and assures that radio communication via $868 \mathrm{MHz}$ and $2.4 \mathrm{GHz}$ is still active even when the microcomputer has shut down or hung up. Connection to the hand controller is made through a trainer-port cable (PPM) (refer to Figure 3) supported by almost all popular remote controllers like FrSky Taranis, Futaba 14SG, Graupner MC-16 etc. HyraCom picks up to 16 channels from the controller. The radio module of the remote controller must be deactivated in order to not interfere with HyraCom's communication.

The touch screen provides a map showing the home point and the aircraft position, as shown in Figure 4. If a GPS is attached, it will be able to show also the pilot's location. Additionally the screen displays basic telemetry from the aircraft as provided by MAVLink telemetry and telemetry of the radio transmission itself (e.g. round trip latencies).

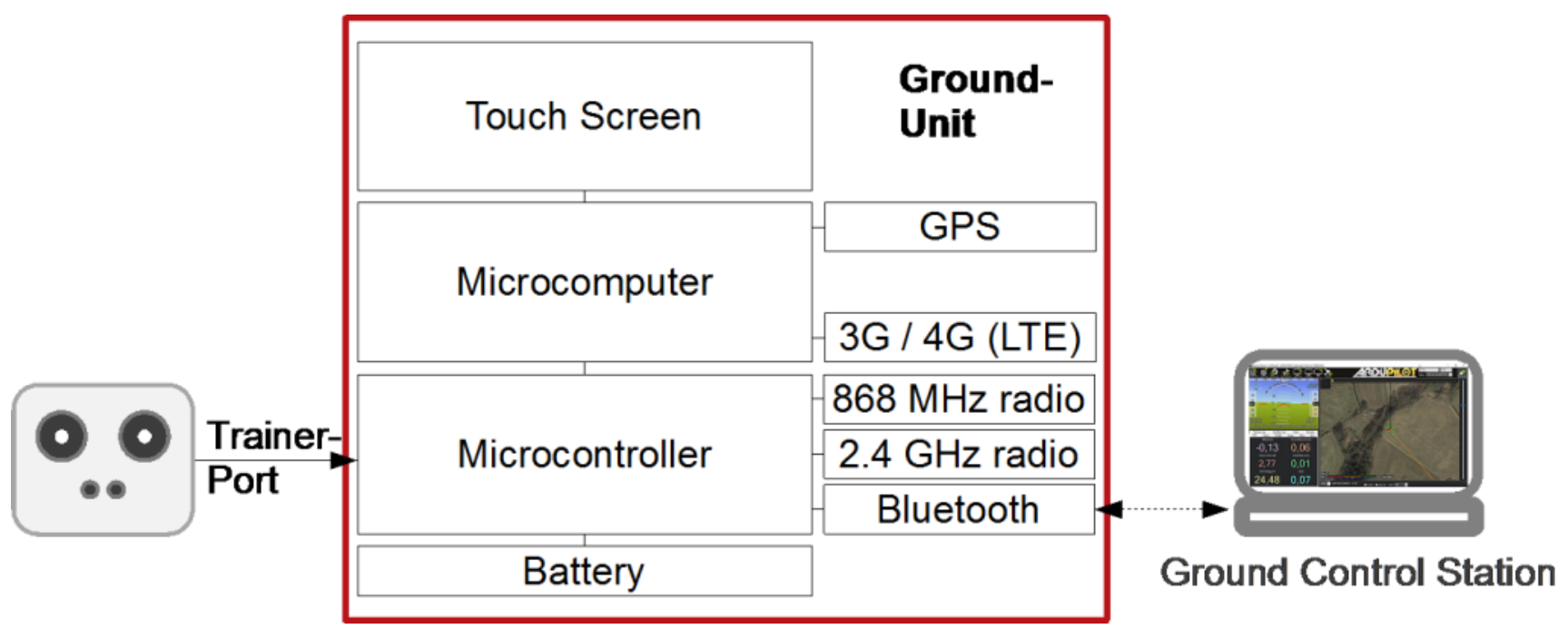

Figure 3 - Ground Unit Components 


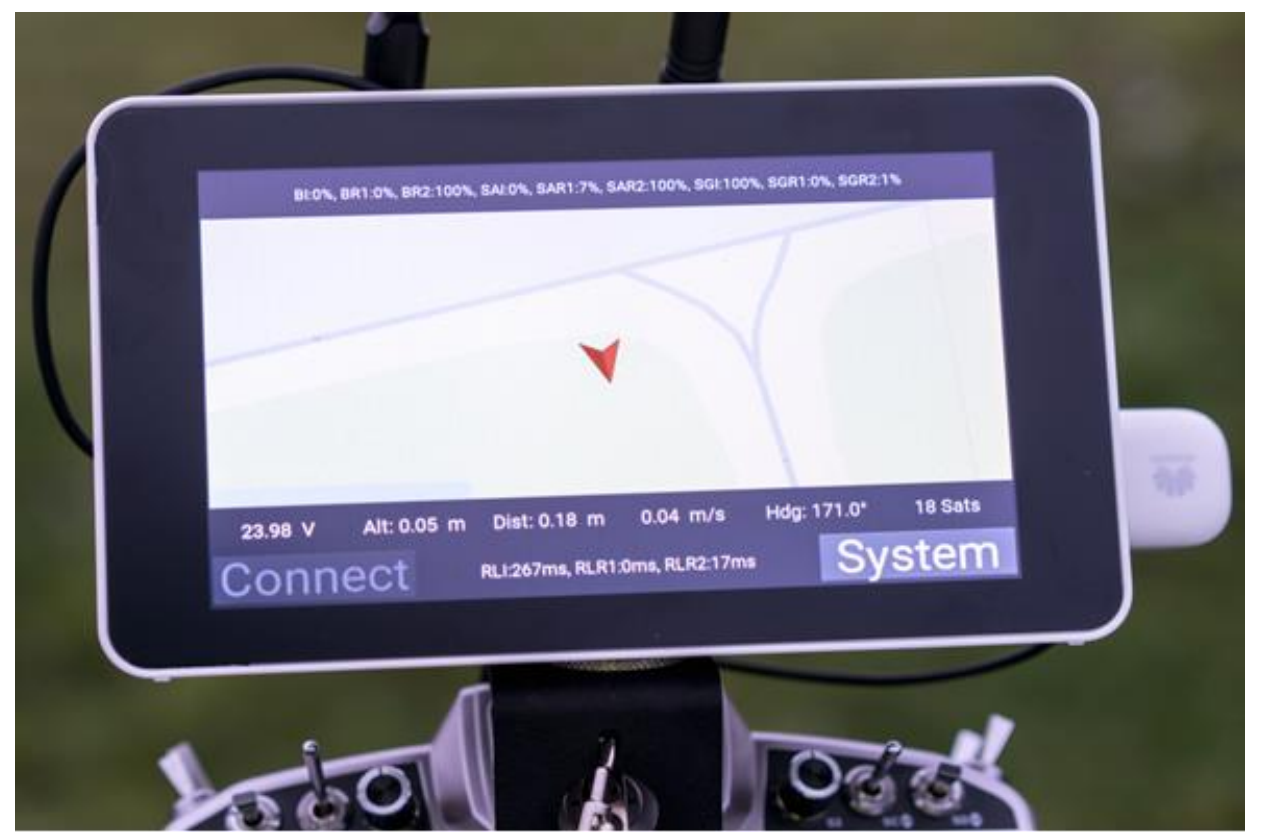

Figure 4 - HyraCom Ground Unit

\section{Air Unit Architecture}

Like the ground unit a microcontroller in the air unit (Figure 5) ensures real-time capability and assures that radio communication via $868 \mathrm{MHz}$ and $2.4 \mathrm{GHz}$ is still possible even when the microcomputer has shut down or hung up. The flight controller will be connected via S-Bus for up to 16 remote control channels. This is supported by almost any flightcontroller like Pixhawk (ArduCopter and
PX4 flight stack), F3 / F4 / F7 (Betaflight, iNav etc.) and DJI A3, N3, Naza V2 etc.

Telemetry and addition commands like waypoint mission upload are transmitted from and to the flight controller through a serial interface $(57600 \mathrm{kbps})$. The transmission is transparent but if the air unit detects MAVLink protocol based telemetry, it applies additional traffic shaping (refer to Figure 6).

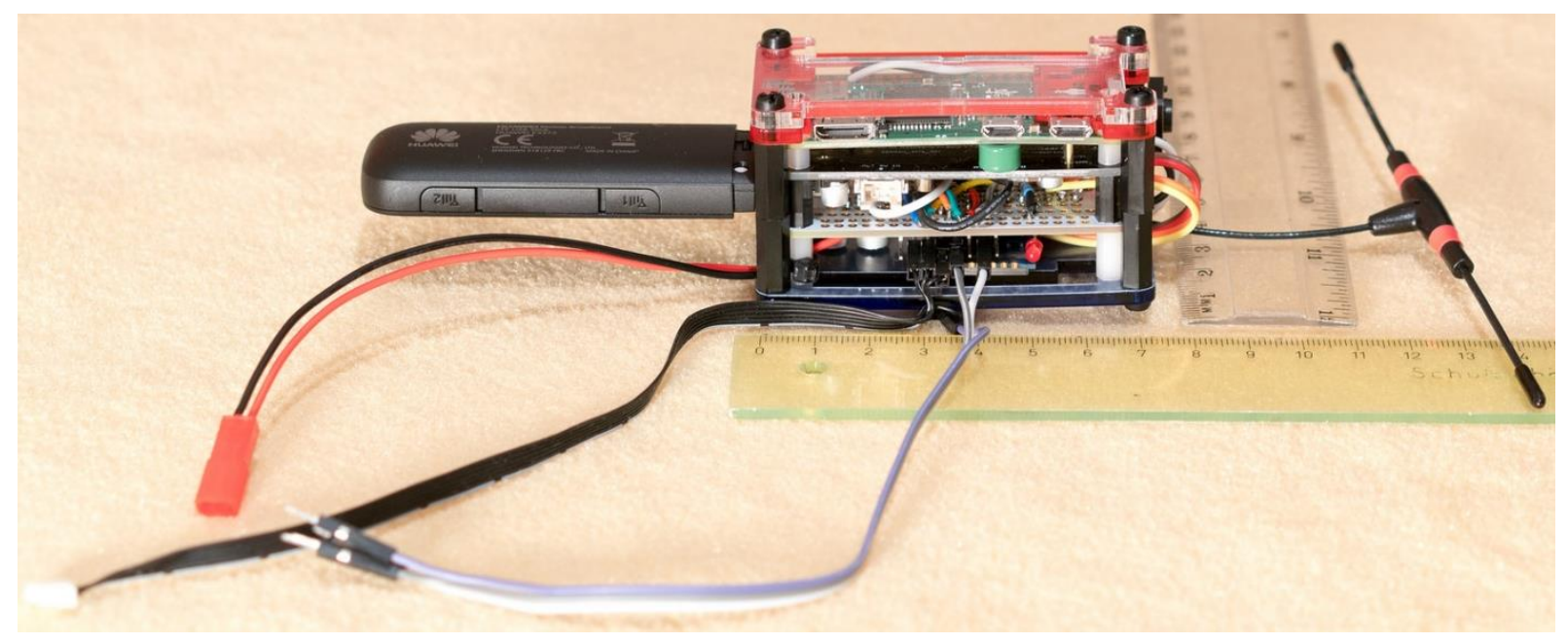

Figure 5 - HyraCom Air Unit 


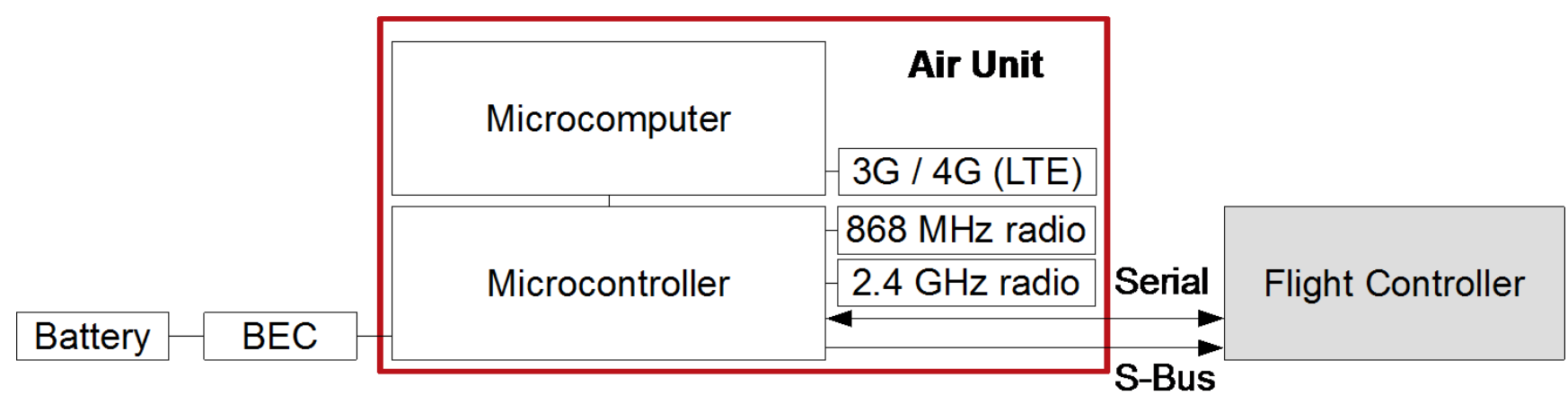

Figure 6 - Air Unit Components

The air unit supports custom made software plugins through an open interface. By default, the air unit contains a reference implementation of a plugin that allows sending UTM_GLOBAL_POSITION messages (\#340) in MAVLink format directly through the mobile network connection to DFS without looping through the ground unit. This makes separate hook-on devices unnecessary.

Alternatively, through this plugin mechanism it is possible to generate UTM_GLOBAL_POSITION messages and transmit them to DFS even when the connected Flightcontroller itself has not implemented this MAVLink message type.

\section{Test Campaign}

All tests will be done with a $10 \mathrm{~m}$ carbon telescope bar and a reinforced cardboard bar extension in order to avoid any disturbances and electromagnetic noise. The Air Unit is usually build in/on a drone, but the structure of the drone, which is usually carbon fiber, electroconductive and therefore possibly shield and disturbs the radio frequencies of the Air Unit's data links. Putting the Air Unit on top of high buildings or towers would have similar effects, since the structure of buildings also contains big metal parts, which shield or damp the intensity of radio frequencies.

Using a telescope bar instead of a drone gives an unlimited air time too, since the flight time of a multicopter drone is limited to its energy source (e.g. fuel tank or battery).

Furthermore noise emission would be a critical issue during the tests if a motor powered drone would be used, since part of the tests are done in urban environments, next to living houses, schools, etc.
The tests not only took place in a rural environment but also in an urban environment because of the chosen 4G LTE frequency from the service providers. In Germany, Telekom uses $862 \mathrm{MHz}$ for 4G LTE Uplinks in rural areas and $1800 \mathrm{MHz}$ in urban areas with a transmitting power of $200 \mathrm{~mW}$ [10]. The $868 \mathrm{MHz}$ radio link of the HyraCom would be heavily disturbed by the rural LTE uplink frequency and reliable measurements would have been impossible [11]. The LTE downlink uses the frequency spectrum of 791 to $821 \mathrm{MHz}$ and is therefore not an issue for the HyraCom.

\section{Test Setup}

The telescope bar can be extended to $10 \mathrm{~m}$, but due to insufficient stability of the last two segments of the telescope bar an altitude of $8 \mathrm{~m}$ was chosen. The cardboard extension gives another $0.5 \mathrm{~m}$ to separate it from the carbon bar. A small carton is attached to the cardboard bar to carry the Air Unit and antennas as shown in Figure 7. The battery is mounted on the carbon bar about $5 \mathrm{~m}$ below the Air Unit for stability reasons and is connected to it via a 5 m USB extension cable. The battery will run the Air Unit for several hours. Figure 8 shows a close-up of the Air Unit and the LiPo battery, which is later attached to the telescope bar later. 


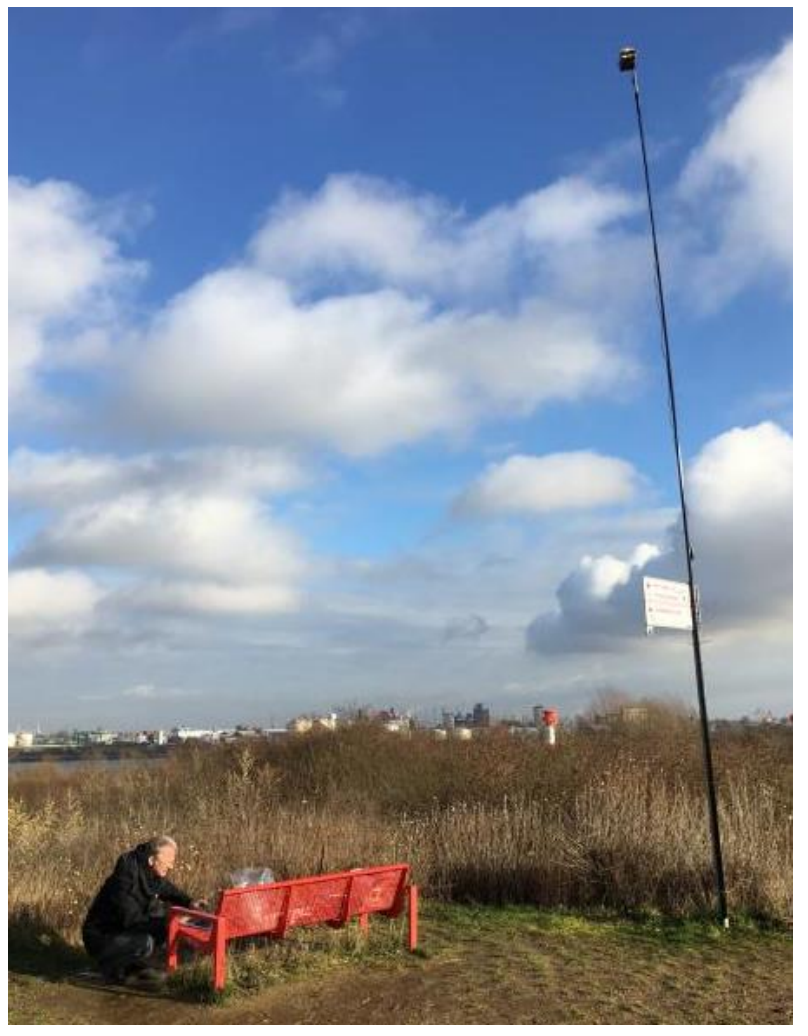

Figure 7 - Cardboard box on telescope bar

To the Air Unit two dipole antennas with linear polarization of the electromagnetic field are attached; for the $868 \mathrm{MHz}$ radio link a $20 \mathrm{~cm}$ dipole antenna with $8 \mathrm{dBi}$, for the $2.4 \mathrm{GHz}$ radio link $16 \mathrm{~cm}$ dipole antenna with $8 \mathrm{dBi}$. The $4 \mathrm{G}$ LTE data link already works with the LTE Stick alone, but is amplified with a $6 \mathrm{~cm}$ folded dipole antenna with $8 \mathrm{dBi}$ with linear polarization of the electromagnetic field. All antennas point straight upwards and stick out of the cardboard box by their full length.

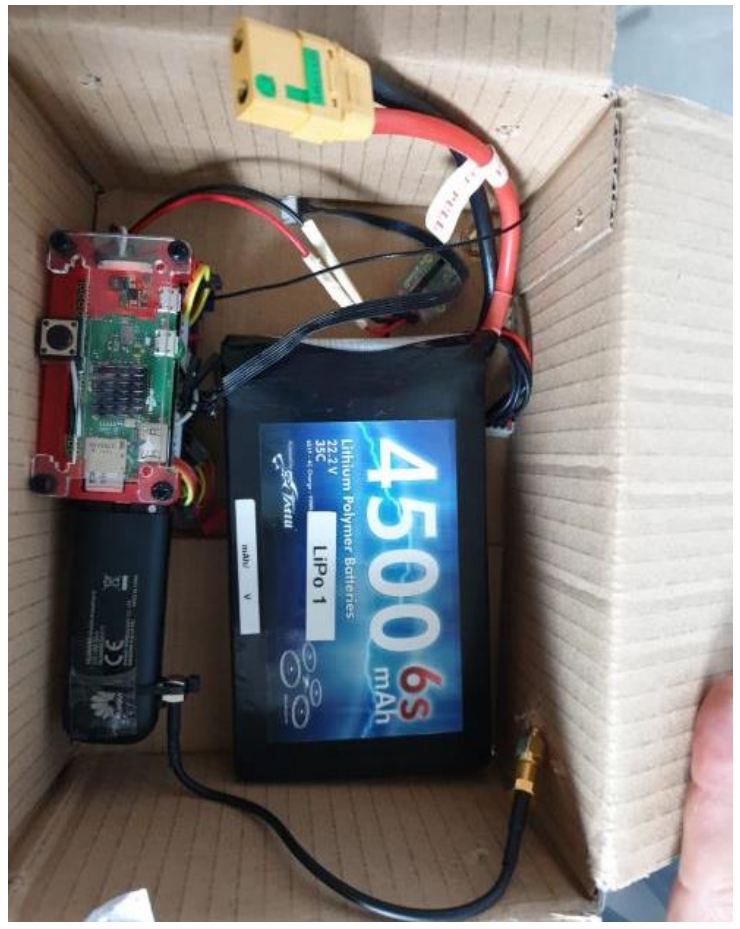

Figure 8 - Air Unit with LiPo battery inside cardboard box

The $868 \mathrm{MHz}$ and the $2.4 \mathrm{GHz}$ radio link each have a repeater to work in a mesh network (Figure 9). A repeater is attached to a camera stand which lifts the repeater to a height of about $2 \mathrm{~m}$ over ground. Both repeaters and a battery are placed in a box and the antennas point straight upwards.

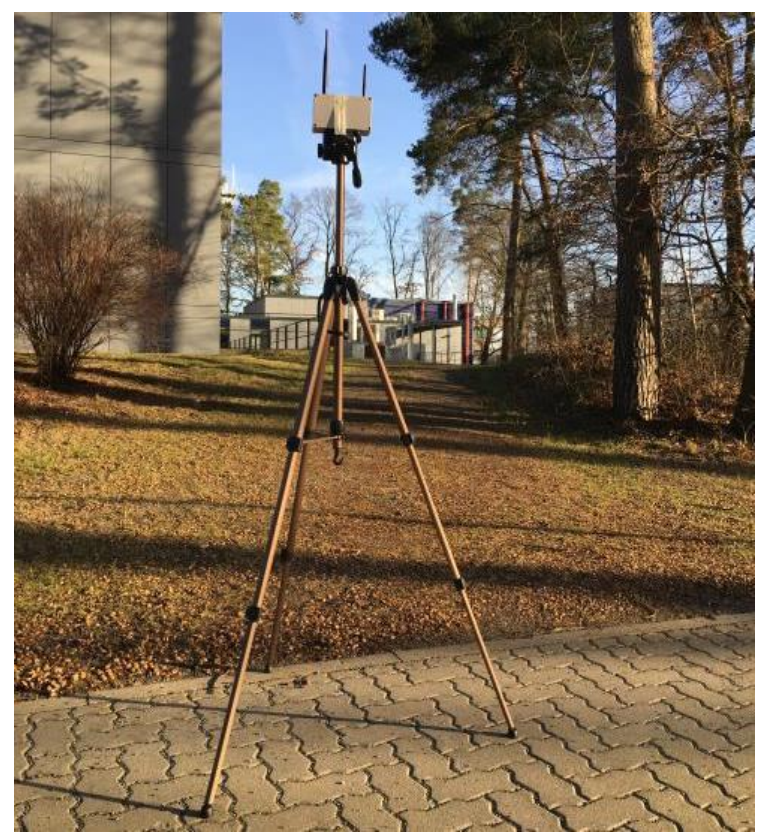

Figure 9 - Repeater on camera stand 
The repeaters are placed well in range of the upraised Air Unit and with direct view to it. This range can only be determined after the maximum range is found out. During all tests regarding the mesh capabilities, the mesh repeater is always closer to the Air Unit than the Ground Unit in order to prevent a bypass of the mesh repeaters.

Note: The repeater was tested in the rural environment only.

For the urban environment a hilly park in Hamburg was chosen, which has an elevation difference of $18 \mathrm{~m}$. The telescope bar with the Air Unit was raised on top of the highest hill (refer to Figure 10). However, the Air Unit was still underneath the tree tops. The Ground Station was carried around in the park by foot.

It can be assumed that the results in the urban environment are more conservative, since more electromagnetic interference and signal-screening infrastructure and objects (e.g. buildings, trees, bushes) are present.

For a rural environment the temporary inoperative airport in Cochstedt/Germany was chosen. It has a runway length of $2.5 \mathrm{~km}$ and is flat without bushes and infrastructure in the way. The telescope bar was raised on the airport fence almost in line with a taxi way; the repeater station was put next to the taxiway in range of the Air Unit. The Ground Station was removed from the Air Unit by car, but exact measurements were made by foot to avoid wrong results by the faraday-ish cage of the car.

\section{Test Procedure}

In the beginning of the tests all devices which are a source of Wi-Fi, Bluetooth, LTE and other disturbing noises are switched off to avoid falsified results. The Air Unit and the Ground Unit are powered; a stable connection of all three data links shall be achieved. In order to avoid a high background noise on the $868 \mathrm{MHz}$ link during the tests in the rural environment, the LTE data link must be terminated since its operating frequency in rural areas is very close to $868 \mathrm{MHz}$. Once all three data links are connected after booting of the systems, both LTE sticks are removed from the Air Unit and Ground Unit respectively; this will terminate the LTE data link. In urban areas this is not an issue and the LTE data link can be left in place and working.

When the Air Unit is raised to $8 \mathrm{~m}$, the Ground Unit is moved away from the Air Unit. The connectivity of the data links as well as the latency of each link is recorded during the whole process, either by data logging or by taking notes. Using data logging the Ground Unit can be moved away from the Air Unit until both radio links are out of range. It can be assumed that the LTE link is never out of range in urban environments (refer to section Results \& Conclusion).

Not using data logging but taking notes is different; the Ground Unit is moved away from the Air Unit in $250 \mathrm{~m}$ increments. The indicated round trip latencies of the three radio links (two radio links in rural area) shall be noted ten times at each distance increment. Since the round trip latency values are quite volatile, an average shall be calculated later. Where one of the radio links terminates (range exceeded), the distance shall be noted. The procedure is continues, until the other radio link terminates.

After these tests the Repeater shall be put in a distance to the Air Unit where both radio links have "good" connectivity, respectively where one radio link has "good" connectivity. The exact distance has to be determined during the tests (refer to section Results \& Conclusion). Afterwards the Ground Unit is moved away from the repeater and the Air Unit. The procedure is the same as described above. 


\section{Results \& Conclusion}

\section{Connectivity in urban environment}

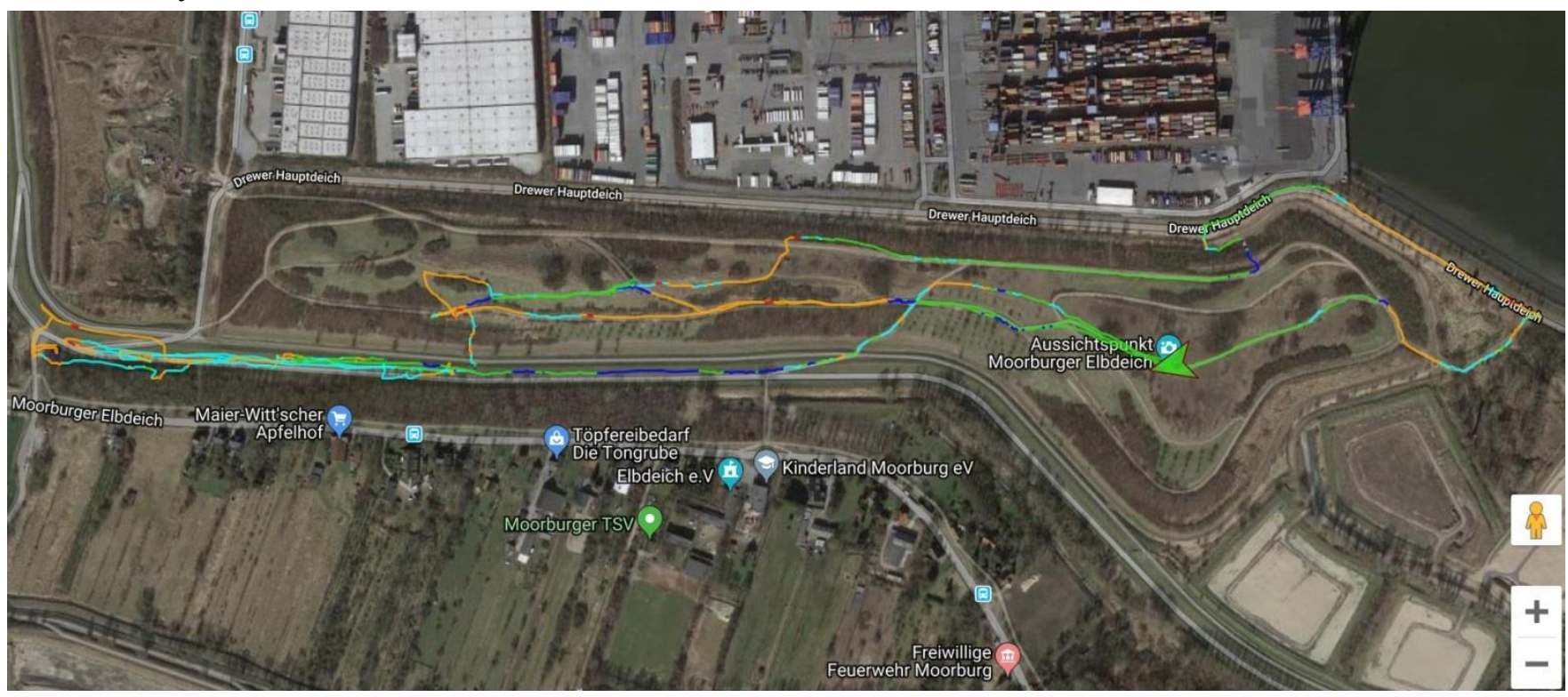

Figure 10 - Connectivity of $868 \mathrm{MHz}, 2.4 \mathrm{GHz}$ and LTE link in urban environment

\footnotetext{
Connectivity on all three radio links

Connectivity on LTE and $2.4 \mathrm{GHz}$

Connectivity on LTE and $868 \mathrm{MHz}$

Connectivity on LTE only

Roundtrip package on all connections lost
}

The day of testing in the park in Hamburg was dry and without fog at $+3^{\circ} \mathrm{C}$. The trees and bushes had no leaves which could hinder the radio links, but the branches were wet. The Air Unit was raised on top of the highest hill (green arrow in Figure 10), but some surrounding trees were still taller than the Air Unit.

Going downhill the $868 \mathrm{MHz}$ and $2.4 \mathrm{GHz}$ links were lost quite quickly since trees and bushes were in the line of sight to the Air Unit. However, the 2.4 $\mathrm{GHz}$ could be regained more often throughout the whole trip, when just the tips of trees and bushes covered the direct view to the Air Unit. This is against expectations, since the $868 \mathrm{MHz}$ has a higher transmitting power (most frequencies $25 \mathrm{~mW}$ ERP (Effective Radiated Power), some channels are limited to 5 and $10 \mathrm{~mW}$ ERP) and a lower frequency, thus usually higher range; it should be regained more often and should also be more stable than the 2.4 GHz. This leads to the conclusion that the $868 \mathrm{MHz}$ radio link is not working as anticipated and must be investigated further.

The highest distances could be achieved on the flat street in the south of the park. The Air Unit was almost in line with the street, which is $18 \mathrm{~m}$ below the hill the Air Unit was raised. Only a few big trees covered the direct view.

Dense trees and bushes have a big effect on the radio distances; this effect will rise when the trees and bushes have leaves. Even though, the units where always connect via LTE. The few small red lines in Figure 10 do not mean that there was no connectivity at all; these are occasions where a roundtrip 
measurement data package was lost. Since the logging program updated only every 5 seconds these small red lines have the length of the distance walked in 5 seconds.

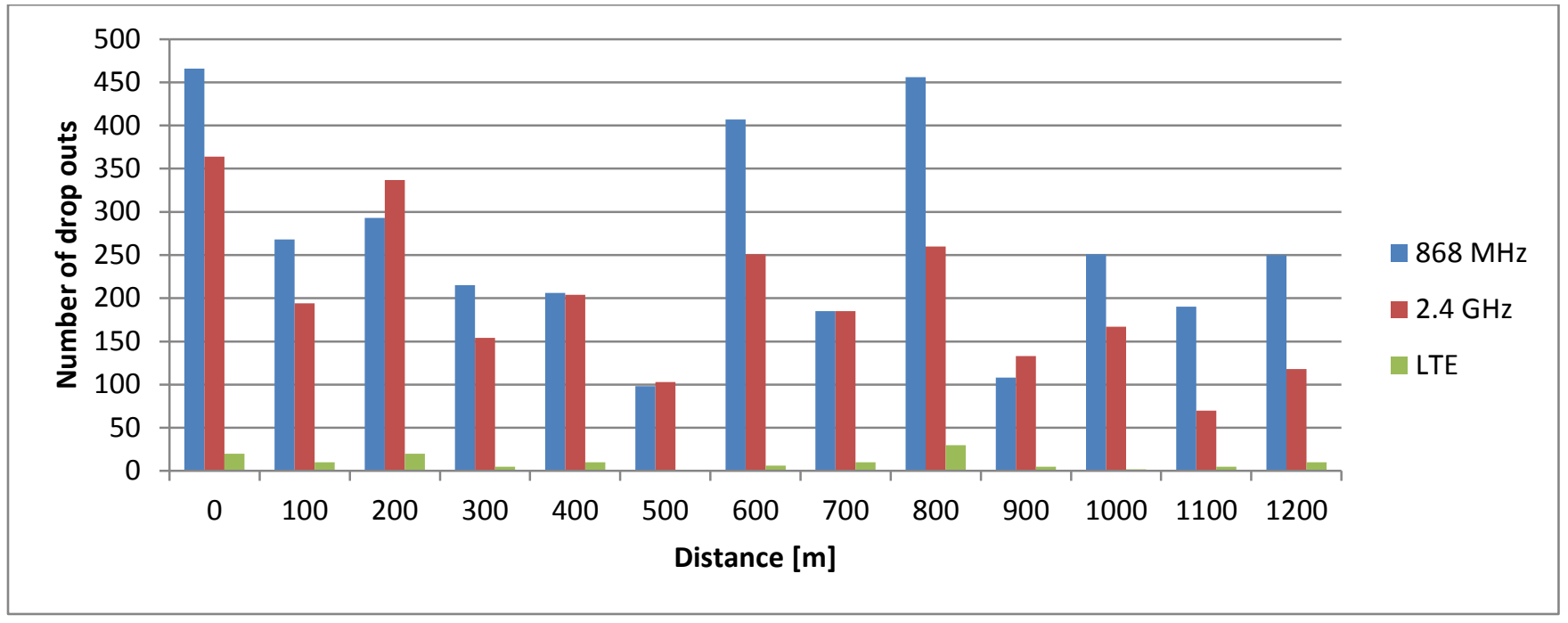

Figure 11 - Number of Drop Outs

Figure 11 underlines the results from Figure 10; the $868 \mathrm{MHz}$ link has the most drop outs, followed by the $2.4 \mathrm{GHz}$ link. The LTE link was very stable apart from a few minor drop outs where a data package was lost.

The number of drop outs is not dependent on the distance from the Air Unit but subject to environmental influences (e.g. trees, bushes, hills).

\section{Latencies in urban environment}

Like the drop outs the latencies are also independent on the distance but are influenced by the environment. The $868 \mathrm{MHz}$ link shows a quite unstable latency trend with RTL (Round Trip
Latency) of 110 to $600 \mathrm{~ms}$. RTL describe the time from the Ground Unit to the Air Unit, processing in the Air Unit and back to the Ground Unit. A pilot would experience only half of the RTL until his control inputs are executed by the drone. Flying larger drones even the $600 \mathrm{~ms}$ RTL would not be noticeable for the drone pilot.

The $2.4 \mathrm{GHz}$ link shows a far more stable latency trend than the $868 \mathrm{MHz}$ link. Also the latencies are much smaller than the $868 \mathrm{MHz}$ link; 22 to $310 \mathrm{~ms}$ RTL.

The LTE link never reaches a latency below 100 ms like the $868 \mathrm{MHz}$ link, but is very stable without much spread with an average RTL of $200 \mathrm{~ms}$.

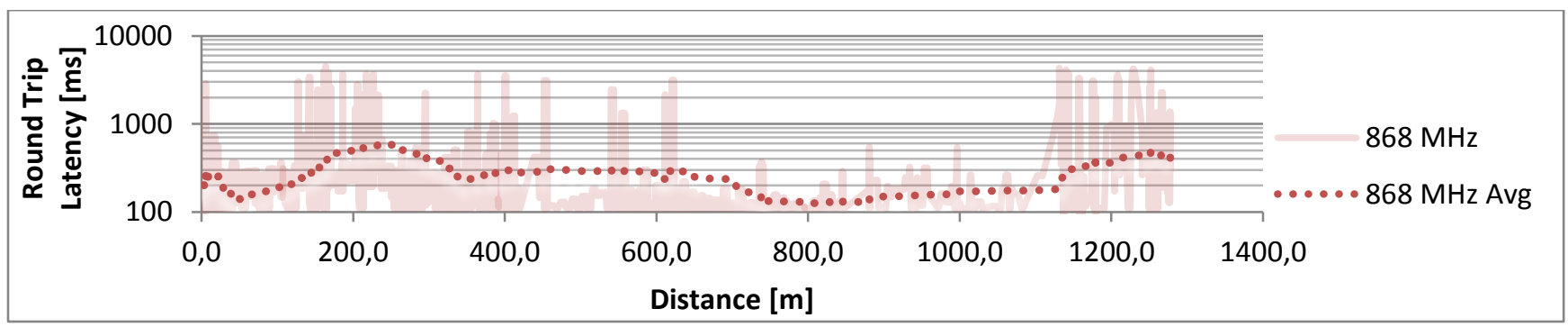



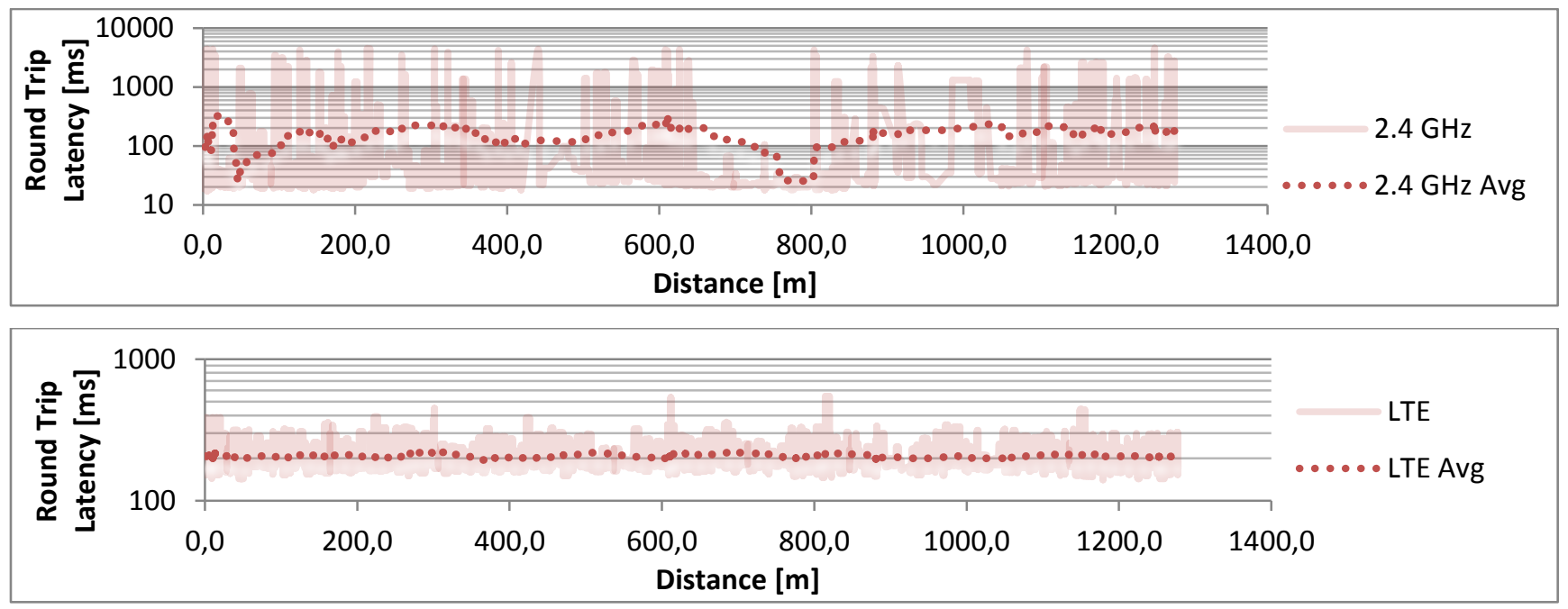

\section{Connectivity in rural environment}

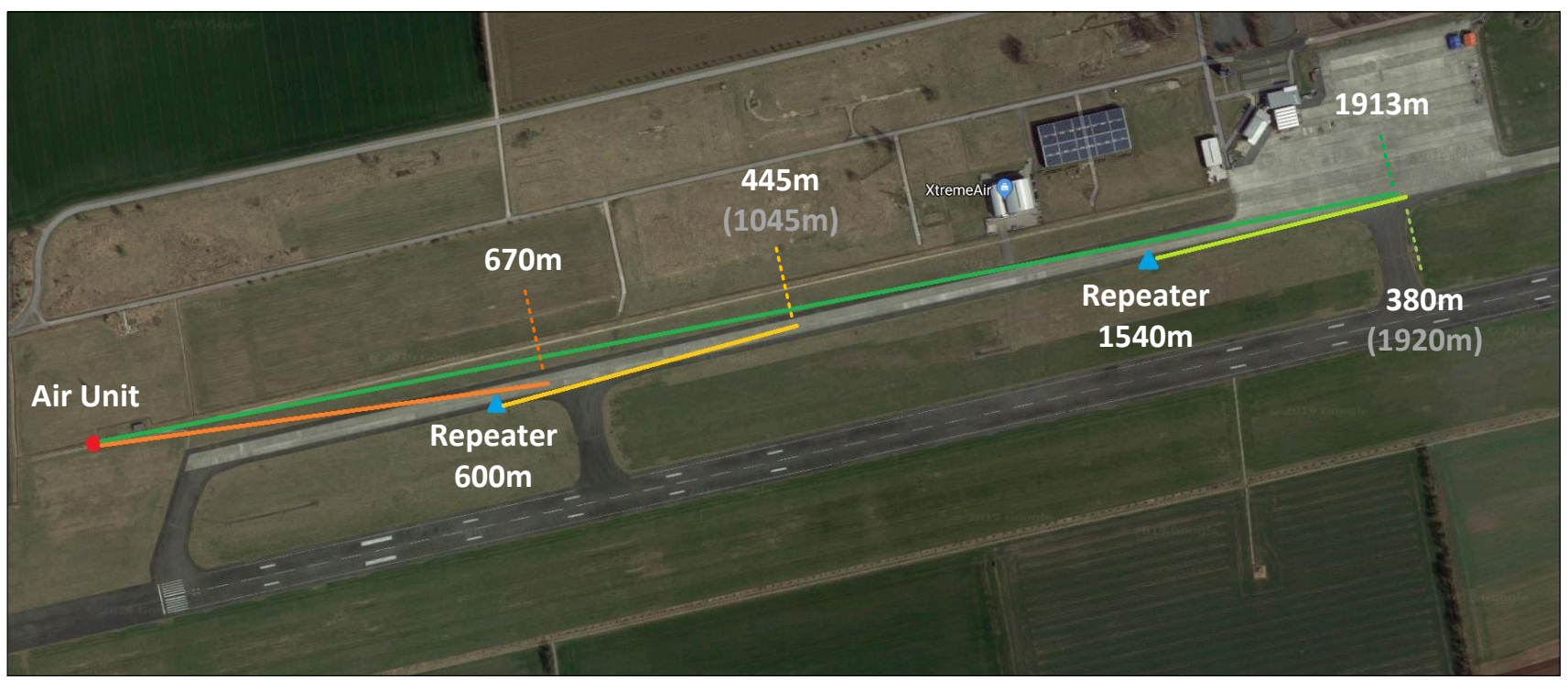

Figure 12 - Connectivity of $868 \mathrm{MHz}, 2.4 \mathrm{GHz}$ and LTE link in rural environment

\subsection{GHz from Air Unit (without Repeater)}

\section{$2.4 \mathrm{GHz}$ via Repeater}

$868 \mathrm{MHz}$ from Air Unit (without Repeater)

$868 \mathrm{MHz}$ via Repeater

The day of testing on the airfield in Cochstedt was dry and without fog at $+5^{\circ} \mathrm{C}$. At no time an obstacle (e.g. building, tree) was in line between the Air Unit, Ground Unit and Repeater.

As it can be seen in Figure 12 the $868 \mathrm{MHz}$ link already terminated after $670 \mathrm{~m}$. The repeater was positioned at $600 \mathrm{~m}$ from the Air Unit. The range from the repeater was $445 \mathrm{~m}$ and it therefore extended the range to $1045 \mathrm{~m}$ in total.

The $2.4 \mathrm{GHz}$ link terminated after $1913 \mathrm{~m}$ without repeater. The repeater was positioned at $1540 \mathrm{~m}$ from the Air Unit. The range from the repeater was $380 \mathrm{~m}$ only and therefore did not extend the range significantly. However, the 2.4 $\mathrm{GHz}$ data link travelled via the repeater, since the overall latency increased considerably during this test. The repeater does currently not seem to add any value to the $2.4 \mathrm{GHz}$, quite the contrary, it increases the latency only. This must be 
investigated further; as the repeater only uses a 2 $\mathrm{dBi}$ dipole antenna, a possible explanation is that the repeater had no connection to the Air Unit anymore but to the Ground Unit. As the Ground Unit still was in range of the Air Unit, the Ground Unit also took over the role of a repeater for the unit that was originally intended to act as repeater. So there was additional work for the Ground Unit but no gain in range.

\section{Latencies in rural environment without repeater}

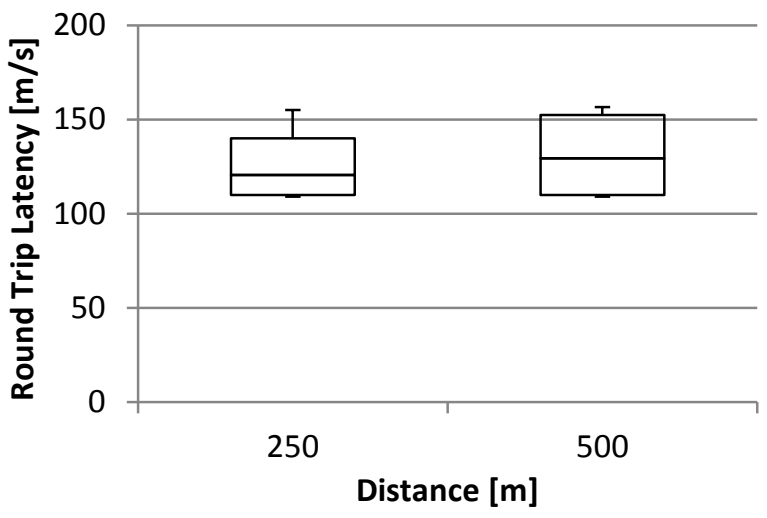

Figure 13 - $868 \mathrm{MHz}$ latency without repeater

The latencies of the $868 \mathrm{MHz}$ link are much more stable in the rural environment than in the urban environment, since no obstacles are in the way. The overall spread is $110 \mathrm{~ms}$ to $155 \mathrm{~ms}$ RTL only (refer to Figure 13). The median ranges from $120 \mathrm{~ms}$ to $130 \mathrm{~ms}$ RTL. In terms of latency the 868 $\mathrm{MHz}$ link shows a much better performance when no obstacles are in the way, thus obstacles decrease the performance of the $868 \mathrm{MHz}$ link significantly.

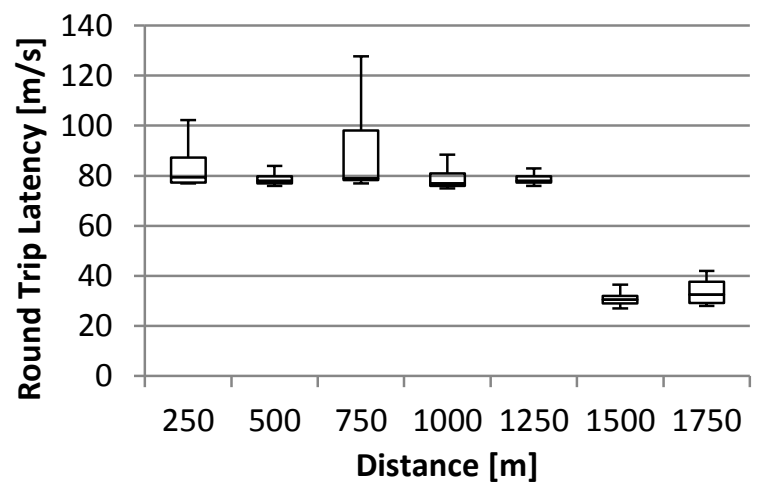

Figure 14 - 2.4 GHz latency without repeater
Like the $868 \mathrm{MHz}$ link the $2.4 \mathrm{GHz}$ shows even better performance without obstacles in the way. The overall spread of the latency is $28 \mathrm{~ms}$ to $98 \mathrm{~ms}$ RTL (refer to Figure 14). The median of the latency is very stable on $80 \mathrm{~ms}$ RTL until the distance of about $1500 \mathrm{~m}$; there the latency median suddenly drops to $30 \mathrm{~ms}$ RTL, which is a significant improvement even when the distance is increased. Why this happens is unclear, since nothing was changed through the testing phase. The runway has a mild elevation increase of $4 \mathrm{~m}$ with distance to the Air Unit, but the author is doubts that this is the cause for this sudden decrease of overall latency.

\section{Latencies in rural environment with repeater}

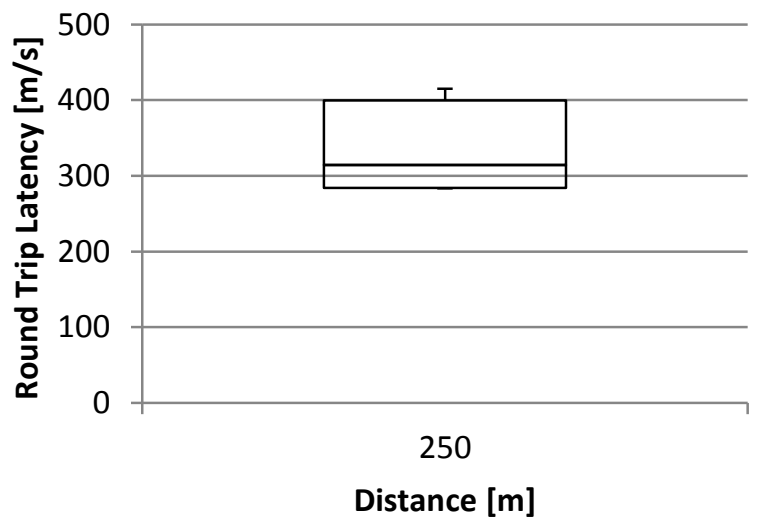

Figure 15 - $868 \mathrm{MHz}$ latency with repeater

With the repeater between the Air Unit and the Ground Unit the latency increases as expected. Also the overall spread increases and ranges from $290 \mathrm{~ms}$ to $400 \mathrm{~ms}$ RTL (refer to Figure 15). The median of the latency is at about $310 \mathrm{~ms}$ RTL which is almost three times as much as without the repeater in rural environment. However, the data recorded for the $868 \mathrm{MHz}$ link with repeater can just give a hint of the behavior; for a reliable predication more data is needed. 


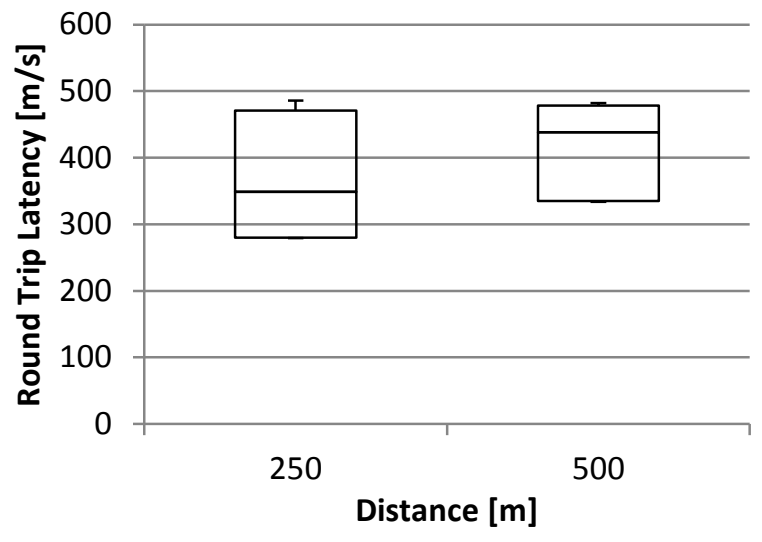

Figure 16 - $2.4 \mathrm{GHz}$ latency with repeater

As expected also the $2.4 \mathrm{GHz}$ link increases in latency. The overall spread increases and ranges from $290 \mathrm{~ms}$ to $480 \mathrm{~ms}$ RTL (refer to Figure 16). The median of the latency from $345 \mathrm{~ms}$ to $440 \mathrm{~ms}$ RTL which is about 5.5 times as much as without the repeater in rural environment. This underlines that the repeater does not work for the $2.4 \mathrm{GHz}$ link as anticipated. Still, flying larger drones the $440 \mathrm{~ms}$ RTL would not be noticeable for a drone pilot.

\section{Outlook}

The results presented in this paper are true for the described communication system only and are not universally valid. There are more systems on the market which provide similar data/radio links; their capabilities should be tested under the same circumstances as described in this paper in order to get a comparison but more important a more holistic view on UAS communication capabilities for a future U-space system.

Additionally the communication systems (including the HyraCom) should be tested on drones in flight since more interference is expected while the system is mounted on a drone due to shielding, carbon fiber influences and onboard electrical systems.

The $868 \mathrm{MHz}$ link must be reviewed overall, since its performance was far under expectations. A fully operating $868 \mathrm{MHz}$ link is a very powerful backup radio link for the drone which could range far distances and therefore ensures a safe operation of drones in BVLOS (Beyond Visual Line of Sight) missions.
Also the repeater needs to be revised since it gave only minor range extension to the $868 \mathrm{MHz}$ link and no range extension at all to the $2.4 \mathrm{GHz}$. If the repeater works as anticipated it could almost double the ranges of both radio links (if no obstacles are in the way). This gives the opportunity for endless scenarios, especially in disaster zones with no or very minor infrastructure.

In order to monitor the temperature of heat emitting units/modules (e.g. engine control unit) temperature sensors (e.g. DS18B20) are connected to the Air Unit to transmit the data to the Ground Unit to provide the drone pilot with the temperature reading.

Also an interface for an optical distance sensor (e.g. LiDAR, single beam) is foreseen for the AirUnit; transmitting the distance to the Ground-Unit in order to monitor the altitude over ground or detect obstacles. This technology also provides the base for the integration of future Detect \& Avoid systems.

For the determination of the drone pilot's position a GPS will be included in the Ground Unit. It provides the opportunity for a dynamic Home Position, but it also can be used for distance measurements from Ground Unit to Air Unit/Drone or for follow-me functions. 


\section{References}

[1] City-ATM, 2019

[https://www.dlr.de/fl/en/desktopdefault.aspx/tabid-

1149/1737_read-50670/].

[2] KopterKraft, 2019

[https://www.kopterkraft.com].

[3] PX4, 2018, Stable Firmware 1.8.2

[https://github.com/PX4/Firmware].

[4] S. Kern, et al. (City-ATM Consortium), 2019, "City-ATM - Demonstration of Traffic Management in Urban Airspace in case of bridge inspection", unpublished.

[5] CORUS (Concept of Operations for European UTM systems), 2019, „Concept of Operations for U-space", unpublished.

[6] Schalk, Lukas Marcel, 2017, "Communication Links for Unmanned Aircraft Systems in Very Low Level Airspace", Integrated Communications Navigation and Surveillance (ICNS) Conference 2017 Integrated Communications Navigation and Surveillance (ICNS) Conference, 18.-20. April 2017, Herndon, VA.

[7] Geister, Dagi and Korn, Bernd, 2018, "Density based Management Concept for Urban Air Traffic", 37th AIAA/IEEE Digital Avionics Systems Conference (DASC) 2018, 23.-27. Sep. 2018, London.

[8] Lieb, Joonas und Peklar, Gerald, 2019, „Evaluation of an unique Communication Interface system D2X for UAVs intercommunicating with Air and Ground UTM users", Integrated Communications Navigation and Surveillance (ICNS) Conference 2019 Integrated Communications Navigation and Surveillance (ICNS) Conference, 09.-11. April 2019, Herndon, VA.

[9] European Telecommunications Standards Institute, 2018, EN 300 220-2, "Short Range Devices (SRD) operating in the frequency range 25 $\mathrm{MHz}$ to $1000 \mathrm{MHz}$ ".

[10] Bundesnetzagentur, 2018, "Frequenzplan" 0 $\mathrm{kHz}$ bis $3000 \mathrm{GHz}$,

[https://www.bundesnetzagentur.de/SharedDocs/Do wnloads/DE/Sachgebiete/Telekommunikation/Unte rnehmen_Institutionen/Frequenzen/Frequenzplan.p $\mathrm{df}$ ?_blob $=$ publicationFile \&v=11].
[11] M. Welpot, S. Wunderlich, I. Gaspard, 2014, „Untersuchung der Störwirkung von LTE auf SRD Anwendungen bei $868 \mathrm{MHz}$ ", Advances in Radio Science, Volume 12, p. 69-74.

2019 Integrated Communications Navigation and Surveillance (ICNS) Conference

April 09-11, 2019 